\title{
Internações e mortalidade por diabetes mellitus na Bahia no período de 2012 a 2018
}

\author{
Hospitalization and mortality due to diabetes \\ mellitus in Bahia in the period of 2012 to 2018
}

\section{Rayanne Rodrigues da Matta Coelho Falcão' ${ }^{1}$ Nathalia Garcia da Silva Santos ${ }^{2}$ (1) Catia Suely Palmeira ${ }^{3}$ (1)}

\begin{abstract}
${ }^{1}$ Autora para correspondência. Escola Bahiana de Medicina e Saúde Pública (Salvador). Bahia, Brasil. rayannerodrigues11@hotmail.com
\end{abstract} 2,3Escola Bahiana de Medicina e Saúde Pública (Salvador). Bahia, Brasil. keilagarciassantos@hotmail.com, catia_palmeira@yahoo.com.br

\begin{abstract}
RESUMO | OBJETIVO: descrever o perfil das internações e da mortalidade por diabetes mellitus na Bahia entre os anos 2012 a 2018. MÉTODO: estudo transversal descritivo realizado com dados secundários oriundos do Sistema de Informações Hospitalares do SUS - SIH/SUS e Sistema de Informações sobre Mortalidade do Ministério da Saúde (SIM/MS). Elegeram-se as variáveis: ano de ocorrência, sexo, faixa etária e caráter de internação. RESULTADOS: no período analisado foram registradas 90510 internações por diabetes mellitus, representando uma taxa de internação de 9,8/10.000 habitantes, 5549 óbitos com taxa de mortalidade de $6,0 \%$. Observou-se crescimento das internações até o ano de 2015 e após este período uma redução. A ocorrência de internações foi maior na faixa etária de 60 a 69 anos (23,7\%), entre as mulheres $(56,2 \%)$, por caráter de urgência $(62,8 \%)$ e na macrorregião Leste $(20,8 \%)$. Quanto à taxa de mortalidade mais elevada foi na macrorregião Leste $(7,3 \%)$, de caráter de urgência $(6,1 \%)$, em pessoas com 80 anos ou mais $(11,9 \%)$ e no sexo masculino $(6,2 \%)$. CONCLUSÃo: o diabetes mellitus configurou-se como importante causa de internação e taxa de mortalidade hospitalar pelos seus números expressivos, principalmente entre os idosos, na macrorregião Leste e que se internam pelo caráter de urgência.
\end{abstract}

DESCRITORES: Internações. Mortalidade. Diabetes Mellitus. Sistema de informação.

\begin{abstract}
OBJECTIVE: to describe the profile of hospitalizations and mortality due to diabetes mellitus in Bahia between the years of 2012 and 2018. METHOD: a crosssectional, descriptive study performed with secondary data from the SUS Hospital Information System (SIH/SUS) and from the Brazilian Ministry of Health's Mortality Information System (SIM/MS). The selected variables were: year of occurrence, gender, age group and nature of hospitalization. RESULTS: during the analyzed period, 90510 hospitalizations were registered for diabetes mellitus, representing a rate of 9.8/10,000 inhabitants, 5549 deaths with a mortality rate of $6.0 \%$. An increase in hospitalizations was observed up until the year 2015, and after that a reduction. The occurrence of hospitalizations was higher for the age group of 60 to 69 years $(23.7 \%)$, among women $(56.2 \%)$, of an emergency nature $(62.8 \%)$ and in the eastern macro-region (20.8\%). In relation to the mortality rate, the highest was in the eastern macro-region (7.3\%), of an emergency nature $(6,1 \%)$, in people of 80 years of age or more $(11.9 \%)$ and of the male gender (6.2\%). CONCLUSION: diabetes mellitus is an important cause of hospitalizations and hospital mortality rates due to its expressive numbers, mainly among the elderly, in the eastern macro-region and hospitalizations of an emergency nature.
\end{abstract}

DESCRIPTORS: Hospitalizations. Mortality. Diabetes Mellitus. Information system. 


\section{Introdução}

A prevalência de diabetes mellitus (DM) está aumentando constantemente em todos os lugares, mais acentuadamente nos países de baixa e média renda. Atualmente cerca de 422 milhões de pessoas em todo o mundo têm diabetes, destacando-se, como uma causa importante de morbimortalidade e um problema de saúde pública ${ }^{1-2}$. Acredita-se, ainda, que aproximadamente $50,0 \%$ dos diabéticos desconhecem que têm a doença, o que impacta no início do tratamento e prevenção da morbimortalidade associada ${ }^{3}$.

Atualmente em âmbito nacional, a doença também representa um problema de saúde de grande magnitude, pois há mais de 13 milhões de pessoas vivendo com diabetes, o que representa $6,9 \%$ da população ${ }^{1}$. Em 2015, por exemplo, aproximadamente $12 \mathrm{mi}-$ Ihões de brasileiros tinham diabetes, e esse número aumentou de 2010 a 2015 em aproximadamente 450.000 casos por ano 4 .

Mundialmente, os custos diretos para o atendimento aos diabéticos variam de $2,5 \%$ a $15 \%$ dos gastos nacionais em saúde, dependendo da prevalência local de diabetes e da complexidade do tratamento disponível ${ }^{5}$. Além dos custos financeiros, a diabetes acarreta também outros custos associados à dor, ansiedade, inconveniência e menor qualidade de vida que afeta os doentes e suas famílias. Representa ainda carga adicional à sociedade, em decorrência da perda de produtividade no trabalho, aposentadoria precoce e mortalidade prematura ${ }^{2}$. As internações por diabetes e complicações dela decorrentes, custam mais caro que internações não relacionadas à doença e suas comorbidades ${ }^{6}$.

O DM consiste em um distúrbio metabólico caracterizado por hiperglicemia persistente, decorrente de deficiência na produção de insulina ou na sua ação, ou em ambos os mecanismos ${ }^{1}$. Destaca-se pelo potencial de desenvolver complicações a longo prazo, sendo as mais comuns no nível macrovascular, como a cardiopatia isquêmica, a doença cerebrovascular e a doença vascular periférica, que muitas vezes levam a morbimortalidade. No nível microvascular, destacase à deficiência visual (retinopatia), doença renal (nefropatia) e dano neuronal (neuropatias), que são as causas mais comuns de cegueira irreversível, doença renal crônica e amputações não traumáticas dos membros inferiores. Isso prova a gravidade do diabetes, pois as complicações relatadas afetam diferentes sistemas do corpo ${ }^{\text {. }}$.

Embora os fatores causais dos principais tipos de DM genéticos, biológicos e ambientais - ainda não estejam completamente conhecidos, os fatores de risco que participam da sua gênese e ao aumento da incidência em todo o mundo, especialmente do diabetes tipo 2, incluem o envelhecimento da população, a crescente prevalência da obesidade, do sedentarismo e os processos de urbanização². A urbanização ocasiona um viver coletivo e o um adensamento populacional que tem relação direta condições de vida e de saúde da população, numa relação de interdependência entre o indivíduo e o meio físico, social e político onde ele vive e se insere ${ }^{8}$.

Apesar do DM, junto com outros agravos crônicos, ser condições consideradas próprias de tratamento no nível de atenção básica $(A B)^{5}$, os quadros agudos e complicações que podem surgir ao longo do tempo, requer assistência em um serviço de natureza hospitalar . As complicações crônicas do diabetes mellitus acarretam prejuízos à capacidade funcional, autonomia e qualidade de vida dos indivíduos em longo prazo, com forte impacto na saúde e finanças dos indivíduos, além de suas famílias e nas economias globalmente ${ }^{2}$.

Assim, a análise das hospitalizações e da mortalidade decorrentes de diabetes mellitus, pode configurar como um indicador de qualidade da assistência prestada pela $A B$, refletindo a efetividade das suas ações e o perfil dos indivíduos internados ${ }^{10}$. Ainda que estudos tenham sido realizados sobre internações e mortalidade por DM em alguns estados e regiões do Brasil, até o momento da realização deste estudo, não haviam sido encontradas publicações com informações específicas da Bahia.

Destaca-se de tal modo, a importância de avaliar dados de internações e mortalidade em uma população, pois tal análise pode subsidiar a proposição de estratégias de prevenção e controle deste agravo, bem como as ações realizadas pelos gestores e profissionais de serviços de saúde neste estado.

Diante disso, questiona-se: qual é o perfil das internações e da mortalidade por diabetes mellitus no estado da Bahia? Para tanto, o presente estudo objetivou descrever o perfil das internações e da mortalidade por diabetes mellitus na Bahia entre os anos 2012 a 2018. 


\section{Método}

Trata-se de um estudo transversal descritivo realizado com dados secundários oriundos do Sistema de Informações Hospitalares do SUS - SIH/SUS e Sistema de Informações sobre Mortalidade do Ministério da Saúde (SIM/MS), gerido pelo Ministério da Saúde através da Secretaria de Assistência à Saúde, em conjunto com as Secretarias Estaduais de Saúde e as Secretarias Municipais de Saúde, processados e disponíveis pelo Departamento de Informática do SUS DATASUS. O levantamento dos dados foi realizado no mês de dezembro de 2019.

O diagnostico principal de internação e causa básica do óbito relacionado ao DM está codificado segundo normas da Classificação Internacional de Doenças em sua Décima Revisão (CID 10), capítulo IV - Doenças endócrinas, nutricionais e metabólicas, na categoria E10 a E14 - Diabetes Mellitus. O Estado da Bahia, o maior da região Nordeste do Brasil, possui um contingente populacional de 14.812.617 habitantes estimados em 2018 , sendo $51,32 \%$ do sexo feminino, um total de 7.601 .009 , e $48,69 \%$ do sexo masculino, ou seja, 7.211.608 habitantes.

A população deste estudo foi composta de todos os casos de internações por diabetes mellitus no estado da Bahia registrados entre 2012 a dezembro de 2018, no Sistema de Informações hospitalares (SIH) e disponível no site do DATASUS. Os dados acerca das internações e mortalidade foram analisados pelo local de residência, segundo ano de ocorrência, sexo, faixa etária, macrorregião, e tipo de internação (eletivo ou urgência).
As taxas de internação foram calculadas pela razão entre o número total de internações por diabetes mellitus de residentes da Bahia pela população total residente estimada pela Fundação Instituto Brasileiro de Geografia e Estatística (IBGE) em 2010, multiplicando-se esse quociente por 10 mil habitantes. A taxa de mortalidade hospitalar foi calculada pela razão entre a quantidade de óbitos e o número de AlH aprovadas, computadas como internações, no período, multiplicada por 100 . Por fim, os dados obtidos foram importados para planilhas do programa Excel de forma a permitir o tratamento descritivo dos dados e posterior análise estatística descritiva.

Quanto aos aspectos éticos, não cabe submissão ao Comitê de Ética em Pesquisa devido à base de dados ser de acesso público seguindo a resolução 466/12 do Conselho Nacional de Ética em Pesquisa.

\section{Resultados}

No período analisado foram registradas 90.510 internações por diabetes mellitus, representando uma taxa de internação de 9,8/10.000 hab. O total de óbitos foi 5.549 e a taxa de mortalidade entre as pessoas internadas para o período correspondeu em média $6,0 \%$. Observa-se um crescimento das internações até o ano de 2015 e após este período uma redução. A maior taxa de mortalidade foi observada em 2017 (7,2\%) (Tabela 1).

Tabela 1. Internações e mortalidade por diabetes mellitus no estado da Bahia, 2012 A 2018

\begin{tabular}{lcccccccc}
\hline Ano & $\mathbf{2 0 1 2}$ & $\mathbf{2 0 1 3}$ & $\mathbf{2 0 1 4}$ & $\mathbf{2 0 1 5}$ & $\mathbf{2 0 1 6}$ & $\mathbf{2 0 1 7}$ & $\mathbf{2 0 1 8}$ & Total \\
Número de internações & 13841 & 14025 & 14936 & 15121 & 11220 & 11386 & 9990 & 90519 \\
Taxa de internação * & 9,8 & 9,9 & 10,5 & 10,7 & 7,9 & 8,0 & 7,1 & - \\
Número de óbitos & 752 & 774 & 843 & 894 & 720 & 817 & 659 & 5459 \\
Taxa de mortalidade ** & 5,4 & 5,5 & 5,6 & 5,9 & 6,4 & 7,2 & 6,1 & - \\
\hline
\end{tabular}

*por 100 casos de internações; ** por 10.000 habitantes

Fonte: Ministério da Saúde - Sistema de Informações Hospitalares do SUS (SIH/SUS) (19/09/2019) 
Os dados na tabela 2 mostram que a maior frequência de internações ocorreu no sexo feminino $(50.884 ; 56,2 \%)$. Em contrapartida o sexo masculino apresentou maior taxa de mortalidade $(6,2 \%)$ quando comparada às mulheres (5,9\%). Com relação ao caráter de atendimento, predominou as internações de urgência (88.945) representando um percentual de $98,3 \%$ do total e com taxa de internação de 62,8/10.000 hab. A mortalidade também foi maior nas internações pelo caráter de urgência, numa razão de 2 para 1 (2:1).

Tabela 2. Distribuição de internações e da taxa de letalidade por diabetes mellitus segundo sexo e caráter de atendimento no estado da Bahia no período de 2012 a 2016

\begin{tabular}{lcccc}
\hline Variáveis & \multicolumn{2}{c}{ Internações } & \multicolumn{2}{c}{ Mortalidade } \\
\cline { 2 - 5 } & N & \% & Óbitos & Taxa (\%) \\
\hline $\begin{array}{l}\text { Sexo } \\
\text { Masculino }\end{array}$ & 39635 & 43,8 & & 6,2 \\
Feminino & 50884 & 56,2 & 2454 & 5,9 \\
Caráter de atendimento & & & 3005 & 3,0 \\
Eletivo & 1574 & 1,1 & & 6,1 \\
Urgência & 88945 & 62,8 & 5412 & \\
\hline
\end{tabular}

* Por 10.000 habitantes

Fonte: Ministério da Saúde - Sistema de Informações Hospitalares do SUS (SIH/SUS) (19/09/2019)

Com relação à faixa etária o maior número de registros de internações ocorreu na faixa de 60 a 69 anos em todos os anos representando $23,7 \%$ do total de internações, seguido pela faixa de 70 a 79 anos (21,7\%). O percentual de internações em menores de dez anos correspondeu a 1,8\% (Tabela 3).

A faixa etária que apresentou maior taxa de letalidade foi a de 80 anos e mais (11,9\%) seguida de 70 a 79 anos (7,36\%). As menores taxas de ocorreram nas faixas de 10 a 19 anos e menores de 10 anos (1,2 \% e 1,3\% respectivamente).

Tabela 3. Internações por diabetes mellitus segundo faixa etária e ano atendimento no estado da Bahia, 2012 A 2018

\begin{tabular}{lcccccccc}
\hline Faixa Etária & $\mathbf{2 0 1 2}$ & $\mathbf{2 0 1 3}$ & $\mathbf{2 0 1 4}$ & $\mathbf{2 0 1 5}$ & $\mathbf{2 0 1 6}$ & $\mathbf{2 0 1 7}$ & $\mathbf{2 0 1 8}$ & Total \\
\hline$<10$ anos & 178 & 201 & 235 & 233 & 243 & 245 & 179 & 1514 \\
10 a 19 anos & 325 & 364 & 391 & 404 & 335 & 379 & 335 & 2533 \\
20 a 29 anos & 414 & 340 & 396 & 380 & 293 & 326 & 270 & 2419 \\
30 a 39 anos & 751 & 691 & 720 & 735 & 550 & 534 & 500 & 4481 \\
40 a 49 anos & 1440 & 1448 & 1554 & 1589 & 1132 & 1140 & 990 & 9293 \\
50 a 59 anos & 2495 & 2563 & 2680 & 2736 & 2062 & 2118 & 1886 & 16540 \\
60 a 69 anos & 3294 & 3420 & 3535 & 3582 & 2681 & 2677 & 2304 & 21493 \\
70 a 79 anos & 2908 & 3082 & 3251 & 3378 & 2404 & 2464 & 2181 & 19668 \\
80 anos e mais & 2036 & 1916 & 2174 & 2151 & 1530 & 1505 & 1345 & 12657 \\
\hline Total & 13841 & 14025 & 14936 & 15121 & 11220 & 11386 & 9990 & 90519
\end{tabular}

Fonte: Ministério da Saúde - Sistema de Informações Hospitalares do SUS (SIH/SUS)(19/09/2019)

A distribuição das internações segundo as macrorregiões mostra que a região Leste e Sul ocupam as duas primeiras posições em todo o período analisado em termos de números absolutos de internações. Quanto à taxa de internação a macrorregião Sul (108,8/100.00 hab.), seguida da macrorregião Centro-Norte (94,0/10.000 hab.) são que apresentam as maiores frequências (Tabela 4). 
Tabela 4. Internações por diabetes mellitus segundo as macrorregiões de residência. Bahia, 2012 a 2018

\begin{tabular}{lcccc}
\hline \multirow{2}{*}{ Macrorregião de Saúde } & \multicolumn{2}{c}{ Internações } & \multicolumn{2}{c}{ Mortalidade } \\
\cline { 2 - 5 } & $\mathbf{n}$ & Taxa* & Óbitos & Taxa (\%) \\
\hline Centro-Leste & 12.213 & 57,6 & 856 & 7,0 \\
Centro- Norte & 7301 & 94,0 & 360 & 4,9 \\
Extremo - Sul & 5413 & 69,8 & 342 & 6,3 \\
Nordeste & 4641 & 56,5 & 333 & 7,1 \\
Norte & 6302 & 61,3 & 393 & 6,4 \\
Leste & 18.872 & 42,6 & 1372 & 7,3 \\
Oeste & 4751 & 54,2 & 195 & 4,2 \\
Sudoeste & 13.405 & 78,3 & 533 & 4,0 \\
Sul & 17.621 & 108,8 & 1075 & 6,4 \\
\hline
\end{tabular}

*10.000 habitantes

Fonte: Ministério da Saúde - Sistema de Informações Hospitalares do SUS (SIH/SUS)(19/09/2019)

\section{Discussão}

Demonstrou-se, neste estudo, que, entre os anos de 2012 a 2015, a tendência de internações por DM na Bahia apresentou um crescimento e após este período uma queda. Com relação a este fenômeno observado não foram encontradas evidências que pudessem explicá-lo, porém, estudo realizado com a população residente do Pará11 encontrou resultados semelhantes: aumento até 2015 e depois redução até 2017, período do estudo. Resultados diferentes foram encontrados em estudos sobre internações por diabetes mellitus no estado do Paraná no período de 2000 a 2012 que apresentou redução de 10,4 para 9,3 internações/10.000 habitantes10 e no Estado de Minas Gerais, de 0,85 para 0,80 internações/1.000 habitantes, en-tre 2000 e 2010 de forma contínua ${ }^{12}$.

Ainda no que concerne ao total de internações, os números encontrados neste estudo (90.519), no período analisado de 7 anos (2012 a 2018) foi maior do que encontrado no estado do Pará em 10 anos, de 2008 a 2007 (53.954), porém a taxa de internação foi menor, pois na Bahia foi de 9,8/10.000 habitantes, enquanto no Pará foi de 71,2/10.000 habitantes ${ }^{11}$.

Segundo os dados da pesquisa telefônica Vigitel 201813 no Brasil, o número de pessoas com diagnóstico de diabetes cresceu $61,8 \%$ em dez anos, portanto era de se esperar que as internações também tivessem um crescimento linear em todo o período analisado. Em 2014, um estudo estimou que um total de 313.273 internações por diabetes ocorreriam no Brasil, correspondendo a 3,6\% do total de internações e representando uma taxa de internação de 22,8 / 10.000 adultos ${ }^{6}$.

Embora o diabetes possa causar diretamente morte e invalidez, a maior parte de sua carga está distribuída entre suas complicações, principalmente doenças cardiovasculares, exigindo abordagens complexas para somar os danos ${ }^{4}$. É importante enfatizar que algumas destas complicações quando se apresentam de forma mais grave, é necessário o tratamento hospitalar. No que tange as complicações advindas da neuropatia, destacam-se o pé diabético e às amputações, com aproximadamente $30 \%$ dos casos anuais no Brasil que necessitam de internação hospitalar ${ }^{14}$.

Neste estudo observou-se que as taxas de mortalidade entre as internações aumentaram até 2017, sendo reduzida em 2018. Corroborando com um estudo que mostrou um aumento na mortalidade por DM no Brasil, nos anos de 1990 a 2015, sendo que em 2015 ocorreu um total de 62.466 mortes por diabetes, sendo $56,1 \%$ mulheres $^{4}$. Para este autor as taxas de mortalidade do Brasil são médias, embora sejam relativamente altas se considerarmos apenas os países da América do Sul.

Estudo brasileiro que avaliou a mortalidade por DM no período de 1996 a 2011 na população brasileira identificou que a maioria $(51,9 \%)$ dos óbitos foi classificado como sem complicações, tendo os outros óbitos como causas específicas de morte às complicações renais ( $19,1 \%$ dos óbitos), circulatórias periféricas ( $6,1 \%$ dos óbitos) e as não especificadas ( $7 \%$ dos óbitos) ${ }^{15}$. 
Ressalta-se ainda que os números sobre a mortalidade podem ser subnotificados, pois, frequentemente, na declaração de óbito, não se menciona o DM, mas sim suas complicações, particularmente as cardiovasculares e cerebrovasculares.

De acordo com Cardozo e Martins16, que realizaram um estudo buscando verificar a existência da correlação entre o envelhecimento e a hereditariedade no desenvolvimento de diabetes mellitus Tipo 2 , os aspectos fisiológicos do envelhecimento contribuem sim para a maior incidência de diabetes nos indivíduos, o que explica o maior número de internações nessa faixa etária.

Com relação a maior frequência de internações ocorreu no sexo feminino. Os resultados encontrados por este estudo estão em concordância com a pesquisa realizada no Ceará17 que também houve predomínio do sexo feminino nas internações por DM $(58,4 \%)$, e pelo estudo realizado na população brasileira $6 \mathrm{em}$ que também se observou um maior número de muIheres internadas pela DM. Ainda neste estudo, os autores analisaram a taxa de internação ajustado pela idade, e encontrou que estas são mais elevadas para nos homens, quando comparadas às mulheres.

Neste estudo a análise das taxas de mortalidade hospitalar mostrou que é maior no sexo masculino. Por um lado, estudo realizado em um município de São Paulo, no que se refere a mortalidade especifica por DM na população geral, verificou-se que a maioria, $321(55,1 \%)$ dos óbitos era de indivíduos do sexo feminino, e predominante na faixa etária maiores de 80 anos de idade. Este aumento expressivo no total de óbitos pode ser observado a partir da faixa etária de 55 anos de idade ${ }^{18}$. Os maiores riscos de óbito nas pessoas mais velhas podem ser decorrentes das fragilidades dos sistemas e órgãos fisiológico própria do envelhecimento e da falta de cuidado adequado $\frac{19}{}$.

Quanto ao caráter de atendimento, predominou as internações e a taxa de mortalidade hospitalar pelo de urgência. Nesta linha, as taxas de hospitalização nessas condições podem também ser resultados da falta conhecimento e de atenção oportuna e efetiva aos problemas de saúde, pelo próprio individuo, que só procura assistência quando a doença já está em um grau mais avançado, ou pela dificuldade de acesso ao serviço de saúde de atenção primária, e sua baixa resolubilidade e efetividade ${ }^{20}$.
A maior proporção de internações na macrorregião leste pode ser explicada pela maior população que reside naquela região. É importante ressaltar as diferenças consideráveis das condições de saúde das macrorregiões, inclusive a quantidade de habitantes entre elas, sendo a Leste a mais populosa, com 4.353.829 habitantes, concentrando $31,7 \%$ do total de habitantes. Esta macrorregião inclui a microrregião de Salvador e zona metropolitana responsável por $72,7 \%$ dos habitantes desta macrorregião21.

Esses dados indicam que a macrorregião leste por englobar a maior e mais desenvolvida cidade do estado, também deve disponibilizar os maiores e mais complexos serviços de saúde hospitalares, para onde confluem pessoas com problemas de saúde mais complexos. Verifica-se grande influência da estruturação dos serviços de saúde em cada território, sobre as hospitalizações com destaque para a capacidade e estrutura do serviço ${ }^{10}$, com melhor capacidade do sistema para diagnóstico e realização de procedimentos mais complexos, às vezes necessários no tratamento das complicações do DM. Os coeficientes de mortalidade hospitalar por DM segundo as macrorregiões apresentaram oscilações para cada uma delas, sendo maior nas macrorregiões Leste Nordeste e Sul e menor na macrorregião Sudoeste.

Convém salientar que estudos que utilizam dados disponíveis no SIH-SUS apresentam algumas limitações: o sistema registra somente as internações realizadas no âmbito do SUS, o que representa cerca de $70 \%$ das internações ocorridas, deixando de fora as hospitalizações nos serviços privados. Além disso, os dados são de difícil análise por causa do preenchimento que pode ser inadequado, pelos possíveis erros de digitação e de registro, e principalmente pela possibilidade de subnotificações. Outra limitação pode ser o curto período de analise de sete anos.

\section{Conclusão}

O presente estudo evidenciou que o diabetes mellitus é importante causa de internação e de taxa de mortalidade das pessoas internadas por esta causa. A maior frequência de registro de internação ocorreu na faixa etária de 60 a 69 anos entre as mulheres. Enquanto que as taxa de mortalidade hospitalar pela causa especifica de DM foi maior entre as pessoas com 80 anos ou mais e entre os homens. 
Os dados permite afirmar que as internações ocorreram principalmente pelo caráter de urgência, inclusive apresentando maiores taxas de mortalidade por esta forma de internação. A macrorregião Leste, onde se situa a cidade mais populosa do estado, sua capital Salvador também foi responsável pela maior frequência de internações e taxas de mortalidade.

Assim, os resultados aqui apresentados corroboram a elevada importância do diabetes mellitus tipo no cenário do estado da Bahia no período de 2012 a 2018. Considerando tais colocações, pensa-se ser importante a realização de mais estudos que incluam mais variáveis de modo a conhecer com profundidade a magnitude do DM neste estado. Tais considerações apontam que o conhecimento das características das internações e mortalidade permitirá o planejamento de ações mais efetivas de controle da doença no nível da atenção básica e melhoria da assistência hospitalar possa reduzir a morbimortalidade do DM.

\section{Contribuições das autoras}

Santos NGS e Falcão RRMC participaram da concepção e delineamento, coleta de dados, redação e encaminhamento do artigo. Palmeira CS participou da busca e análise estatísticas dos dados da pesquisa, interpretação dos resultados, redação do artigo científico.

\section{Conflitos de interesses}

Nenhum conflito financeiro, legal ou político envolvendo terceiros (governo, empresas e fundações privadas, etc.) foi declarado para nenhum aspecto do trabalho submetido (incluindo, mas não se limitando a subvenções e financiamentos, participação em conselho consultivo, desenho de estudo, preparação de manuscrito, análise estatística, etc.).

\section{Referências}

1. Oliveira JEP, Montenegro Junior RM, Vencio S, organizadores. Diretrizes da Sociedade Brasileira de Diabetes 2017-2018. São Paulo: Editora Clannad; 2017.

2. World Health Organization - WHO. Fact sheet on diabetes. [Internet]. 2018. [acesso em 31 de out. 2018]. Disponível em: https://www.who.int/news-room/fact-sheets/detail/diabetes

3. Beagley J, Guariguata L, Weil C, Motala AA. Global estimates of undiagnosed diabetes in adults. Diabetes Res Clin Pract. 2014;103(2):150. doi: 10.1016/j.diabres.2013.11.001
4. Duncan BB, Schmidt MI, Cousin E, Moradi-Lakeh M, Passos VMA, França EB et al. The burden of diabetes and hyperglycemia in Brazil-past and present: findings from the Global Burden of Disease Study 2015. Diabetol Metab Syndr. 2017;9:18. doi: 10.1186/s13098-017-0216-2

5. Saraiva JFK, Hissa MN, Felício JS, Cavalcanti CAJ, Saraiva GL, Piha T et al. Diabetes mellitus no Brasil: características clínicas, padrão de tratamento e custos associados ao cuidado da doença. J Bras Econ Saúde. 2016; 8(2):80-90. doi: 10.21115/JBES.v8.n2.p80-90

6. Rosa MQM, Rosa RDS, Correia MG, Araujo DV, Bahia LR, Toscano CM. Disease and Economic Burden of Hospitalizations Attributable to Diabetes Mellitus and Its Complications: A Nationwide Study in Brazil. Int J Environ Res Public Health. 2018; 15(2). doi: 10.3390/ijerph15020294

7. Silva EFF, Ferreira CMM, Pinho L. Risk factors and complications in type 2 diabetes outpatients. Rev Assoc Med Bras. 2017;63(7):621-7. doi: 10.1590/1806-9282.63.07.621

8. Kondo MC, South EC, Branas CC. Nature-based Strategies for Improving Urban Health and Safety", in Journal of Urban Health. J Urban Health. 2015;92(5):800-14. doi: 10.1007/s11524-015-9983-y

9. Cortez DN, Reis IA, Souza DAS, Macedo MML, Torres HC. Complications and the time of diagnosis of diabetes mellitus in primary care. Acta paul. Enferm. 2015;28(3):250-255. doi: 10.1590/1982-0194201500042

10. Arruda GO, Schmidt DB, Marcon SS. Hospitalizations for diabetes mellitus and the Family Health Strategy, Paraná, Brazil, 2000-2012. Ciênc saúde coletiva. 2018;23(2): 543-52. doi: 10.1590/1413-81232018232.23092015

11. Araujo CC, Cunha CLF, Valois RC, Botelho EP, Barbosa JS, Ferreira GRON. Internações por diabetes mellitus no estado do Pará: distribuição espacial e fatores associados ao óbito. Rev Nursing. 2019; 22(257):3226-33.

12. Rodrigues-Bastos RM, Campos EMS, Ribeiro LC, BastosFilho MG, Bustamante-Teixeira MT. Internações por condições sensíveis à atenção primária, Minas Ge $\neg$ rais, 2000 e 2010. Rev Saúde Publica 2014;48(6):958-67. doi: 10.1590/ S00348910.2014048005232

13. Ministério da Saúde. Secretaria de Vigilância em Saúde. Departamento de Análise em Saúde e Vigilância de Doenças não Transmissíveis. Brasil: Vigilância de fatores de risco e proteção para doenças crônicas por inquérito telefônico: estimativas sobre frequência e distribuição sociodemográfica de fatores de risco e proteção para doenças crônicas nas capitais dos 26 estados brasileiros e no Distrito Federal em 2018. Brasília: Ministério da Saúde; 2019.

14. Costa AF, Flor LS, Campos MR, Oliveira AF, Costa MFS, Silva RS et al . Carga do diabetes mellitus tipo 2 no Brasil. Cad. Saúde Pública. 2017;33(2):e00197915. doi: 10.1590/0102-311×00197915 
15. Schmidt MI, Duncan BB, Ishitani L, Franco GC, Abreu DM, Lana GC et al. Trends in mortality due to diabetes in Brazil, 1996-2011. Diabetol Metab Syndr. 2015;7:109. doi: 10.1186/S13098-015-0105-5

16. Cardozo ACO, Martins CRAL. Correlação Entre o

Envelhecimento e a Hereditariedade no Desenvolvimento de Diabetes Mellitus Tipo 2: Um Estudo Transversal Analítico. Revista de Divulgação Científica Sena Aires. 2012;1(2):133-140.

17. Santos FAL, Lima WP, Santos AL, Teston EF, Marcon SS. Hospitalizações por diabetes em adultos e idosos no Ceará, 20012012. Epidemiol Serv Saúde. 2014;23(4):655-63. doi: 10.5123/ $\underline{\mathrm{S} 1679-49742014000400007}$

18. Lima RAD, Istilli PT, Teixeira CRS, Zanetti ML, Torquato MTCG. Diabetes mellitus mortality in a municipality in the state of São Paulo, 2010 to 2014. Rev Saude Publica. 2019; 53(24):1-9. doi: 10.11606/S1518-8787.2019053000561
19. Antunes JFS, Okuno MFP, Lopes MCBT, Campanharo CRV, Batista REA. Frailty assessment of elderly hospitalized at an emergency service of a university hospital. Cogitare Enferm. 2015;20(2):264-71.

20. Avelino CCV, Goyatá SLT, Nogueira DA, Rodrigues LBB, Siqueira SMS. Qualidade da atenção primária à saúde: uma análise segundo as internações evitáveis em um município de Minas Gerais, Brasil. Ciênc saúde coletiva. 2015;20(4):1285-93. doi: 10.1590/1413-81232015204.12382014

21. Secretaria de Saúde do Estado da Bahia - SESAB. Plano Estadual de Educação Permanente em Saúde da Bahia. Governo do Estado da Bahia.[Internet]. 2018. Disponível em: https://www. conass.org.br/planos-estaduais-educacao-permanente/PEEPS-BA. pdf 